\title{
Stokes parameters determination in LWIR spectrum with imaging IR camera setup
}

\author{
by G. Bieszczad, M. Łoniewski and S. Gogler
}

Military Univ. of Technology, 00-908, gen. S. Kaliskieg Str., Warsaw, Poland, grzegorz.bieszczad@wat.edu.pl

\begin{abstract}
Most frequently used thermal imaging devices respond only to one physical quantity, that is radiance. In more sophisticated devices, the waveband of concern is split into sub-bands and energy flux is quantified per sub-band. These are so-called multispectral or hyperspectral cameras and are used for detection and discrimination of chemical compounds, very frequently hazardous gases. Even in these devices however only one physical quantity is analysed spectral radiance. It has been shown that the analysis of radiation against the state of polarization and its spatial distribution across the scene can deliver additional information on observed objects. In the article a measurement stand for determination of polarization state is described and the polarization image properties for chosen objects are described.
\end{abstract}

\section{Introduction}

Imaging polarimetry in long wave infrared spectrum has been a subject of research in leading scientific [1, 2, 3, 5] research centers around the world for a number of years. Across almost entire electromagnetic spectrum imaging polarimetry has found numerous applications including investigation of plant vegetation, satellite research of Earth's atmosphere [4], examining oceans' salinity [6], medical diagnostics for cancer cell identification [8] biomedicine [9], military target detection and search and rescue operations [1, 3, 9].

Principle of operation for polarimetric detection is based on energy partition between different polarization states upon reflection off optical medium discontinuity. If the incidence of radiation on a boundary is non-normal, partial polarization of reflected wave takes place. Typically polarized glasses are used to suppress reflected polarized glare (possibly blinding) and thus increase visibility. The principle is the same in infrared electromagnetic radiation range and detection of polarized radiation can bring additional information about observed scene. For example a naturally-occurring objects are texturized and light reflected off their surface is mostly unpolarized while artificial objects are often smooth and present polarimetric signatures. This property can be exploited to distinguish natural objects from hidden artificial objects. Sometimes there may be the opposite situation when polarized light comes from the smooth surface of the water, and the unpolarized from the object (e.g. a survivor) swimming on its surface.

Polarized radiation is characterized by wide dynamic range and large spatial variations. Measured polarimetric information depends heavily on mutual geometry of the scene, source and detector and so measured signal changes accordingly to daytime, localization and detector orientation.

In the article a measurement stand for determination of polarization properties of several various objects is described. The method for polarimetric characterization is shown along with measurement setup comprising cooled infrared camera, external polarizing filter, technical black bodies in elaborated geometry and algorithm for infrared signal processing and calibration. Additionally a method for polarization state visualization is also described.

\section{Polarization state imaging}

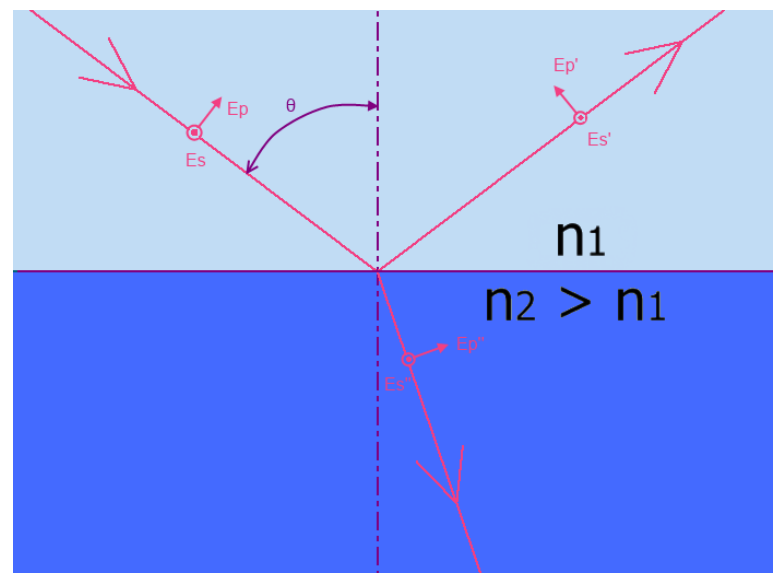

Fig. 1. Light reflection and refraction at medium boundary 


\subsection{1/qirt.2016.008}

The purpose of the experiment was to determine polarimetric signatures and properties of commonly found objects. Polarization signature determination can be then useful in defining demands for remote object detection systems with polarization state analysis. In experiment, the polarization state signatures in Long-Wave Infrared spectrum were induced by external irradiation and its reflection from specimen surface. Polarization of light upon reflection at a medium discontinuity is described with Fresnel [10] equations:

$$
r_{\mathrm{S}}=\frac{n_{1} \cos \theta-n_{2} \sqrt{1-\left(\frac{n_{1}}{n_{2}} \sin \theta\right)}}{n_{1} \cos \theta+n_{2} \sqrt{1-\left(\frac{n_{1}}{n_{2}} \sin \theta\right)}}, r_{p}=\frac{n_{1} \sqrt{1-\left(\frac{n_{1}}{n_{2}} \sin \theta\right)}-n_{2} \cos \theta}{n_{1} \sqrt{1-\left(\frac{n_{1}}{n_{2}} \sin \theta\right)}+n_{2} \cos \theta}
$$

where $r_{\mathrm{s}}$ and $r_{p}$ are amplitude reflection coefficients for a plane monochromatic wave with electric field vector perpendicular ( $s$ for german senkrecht) and parallel $(p)$ to the plane of incidence (plane of this sheet in figure below). Reflected amplitude for a given component is given by:

$$
E_{s}^{\prime}=E_{s} r_{s}, \quad E_{p}^{\prime}=E_{p} r_{p}
$$

and reflected power is then:

$$
I_{s}^{\prime}=E_{s} E_{s}^{*} \cdot r_{s} r_{s}^{*}=I_{s} R_{s}, \quad I_{p}^{\prime}=E_{p} E_{p}^{*} \cdot r_{p} r_{p}^{*}=I_{p} R_{p}
$$

By using a linear polarizer (polarizing filter) one can filter out radiation with definite plane of vibration. One of commonly used in this field method of description of polarized light is the Stokes vector. Since the quantities used to formulate the vector are measured intensity values, it is a convenient and straightforward method to describe polarization state of light. The Stokes vector is defined as follows:

$$
\left[\begin{array}{l}
S_{0} \\
S_{1} \\
S_{2} \\
S_{3}
\end{array}\right] \stackrel{\text { def }}{=}\left[\begin{array}{c}
\left\langle\left|E_{S}\right|^{2}+\left|E_{p}\right|^{2}\right\rangle \\
\left\langle\left|E_{S}\right|^{2}-\left|E_{p}\right|^{2}\right\rangle \\
2 \operatorname{Re}\left\langle E_{S} * \overline{E_{p}}\right\rangle \\
-2 \operatorname{Im}\left\langle E_{S} * \overline{E_{p}}\right\rangle
\end{array}\right] \propto\left[\begin{array}{c}
I_{0}+I_{90} \\
I_{0}-I_{90} \\
I_{45}-I_{135} \\
I_{L}-I_{P}
\end{array}\right]
$$

$\langle E\rangle$ denotes time-average on a timescale much longer than wave period and * denotes complex conjugate. So is the total measured intensity, $S_{1}$ is the intensity of horizontal or vertical polarization, $S_{2}$ is the intensity of linear polarization at an angle of \pm 45 degrees and $S_{3}$ is amount of elliptical polarization (left or right-handed). First three parameters are easily measured with linear polarizer.

To measure the last parameter it is necessary to use a phase retarder (i.e. quarter-wave plate). Since it has been shown [1, 2] that contribution of circularly polarized light is negligible, the costs of the phase retarder for LWIR range outweigh the benefits of that additional measurement. Knowing the parameters $S_{0}, S_{1}$ and $S_{2}$ alone enables one to discriminate objects with regard to state of polarization of radiation reflected from their surface. From measured intensity values given above degree of linear polarization can be estimated and the azimuth of the polarization ellipse:

$$
\begin{aligned}
& \text { DOLP }=\frac{\sqrt{S_{1}^{2}+S_{2}^{2}}}{S_{0}} \\
& \psi=\frac{1}{2} \tan ^{-1}\left(\frac{S_{2}}{S_{1}}\right)
\end{aligned}
$$

Using infrared camera and polarizing filter large enough to cover aperture of cameras' optical lens there is a possibility to determine polarization state of radiation from observed scene and evaluate polarimetric signatures of observed objects. Calculating Stokes parameters of radiation reaching every detector in cameras focal plane array can bring an image of polarization state across observed objects. In the article a measurement stand for determination of polarization properties of several various objects is described.

\section{Experimental setup and procedure}

The aim of the experiment was to determine polarimetric signatures of chosen objects and evaluate polarimetric imaging for detection of man-made objects. Developed measurement stand consists of:

three black bodies,

○ SR-800A-R 20" absolute Blackbody,

- SR-800D 14" Differantial Chamber Blackbody, 
○ SR-800D 14" Differantial Chamber Blackbody,

optical table,

rotary table with a post,

germanium reference piece,

thermal camera FLIR SC7900VL,

$>$ PC with FLIR Altair Software,

$>$ polarizing filter (Image Quality SPECAC 57068)

$>$ black bodies controllers.

Examined specimen was placed inside three blackbody geometry ensuring controlled environment radiation. A picture of the measurement stand is shown in figure 2 and the schematic of the stand is shown in figure 3 .

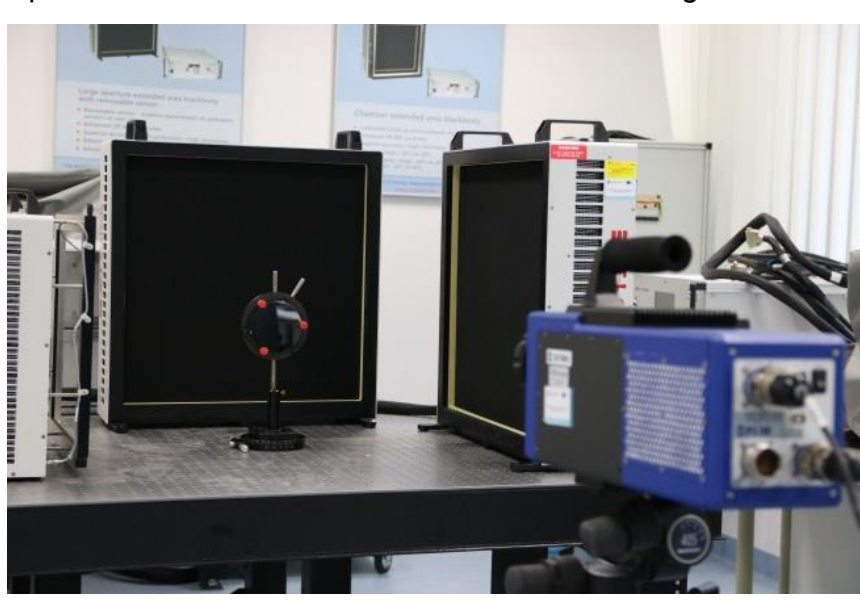

Fig. 2. Photos of measurement stand

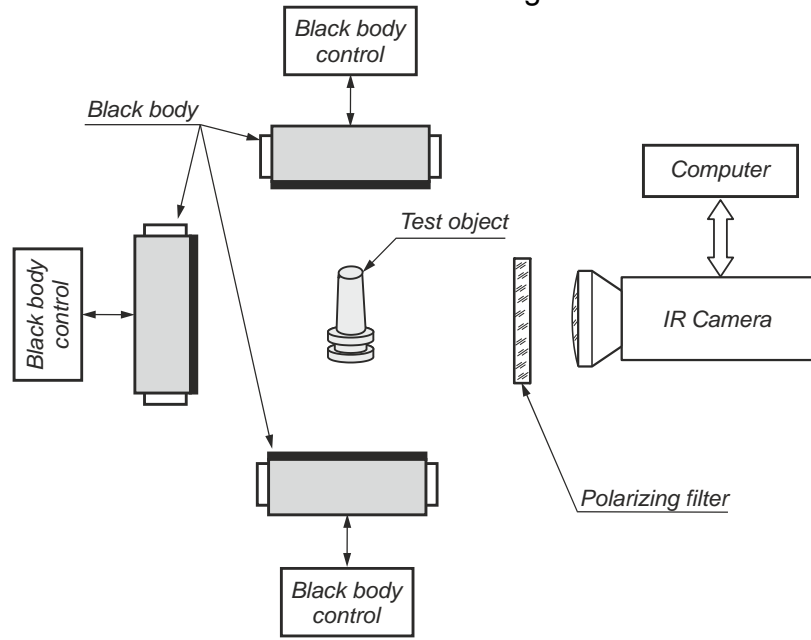

Fig. 3. Diagram of measurement stand

Most straightforward method of polarimetric imaging has been employed i.e. division of time. A series of images has been taken with polarizing filter oriented at different angles and polarimetric data has been calculated in postprocessing step.

\subsection{Calibration precedure}

Calibration procedure for the measurement included:

$>$ standard NUC

$>$ radiometric calibration with polarizing filter in the optical path

$>$ geometric calibration

For best results the NUC was of two-point kind. At the same time internal radiometric camera calibration took place. Since a large black body filling whole aperture and field-of-view is presented to the camera it is a radiance calibration. Altair software was used to perform the radiometric calibration process, non-uniformity correction and to identify and replace defective pixels. Provided software enabled carrying out non-uniformity correction and bad pixel replacement procedures automatically. Because of additional element - polarizer plate - present in the optical path, there was a need to perform manual radiometric calibration of measurement setup. Calibration was performed with use of reference black bodies set to five different temperatures. Registration of cameras' digital level output and assignment to corresponding black body temperature allowed to determine the calibration curve taking into account additional polarizing element. Calibration curve introduced to Altair software, provided radiometrically accurate registration of incident radiation from observed scene. Process of radiometric calibration and non-uniformity correction carried out in Altair software is presented on figure 6 . Rotation of polarizer plate during registration introduced a requirement for additional non-uniformity correction and was performed before every image registration with polarizing filter.

Since evaluation of Stokes parameters is based on intensity measurements usually extinction ratios of analyzing elements have to be known:

$$
I_{m}(\theta)=\gamma \frac{\boldsymbol{p}}{2}[1 \cos (2 \theta) \sin (2 \theta) 0]\left(\boldsymbol{S} I_{i}\right)
$$

$I_{m}(\theta)$ is measured intensity as a function of polarizer rotation $\gamma$ is calibration factor, $p$ is extinction ratio $S$ the Stokes vector an $I_{i}$ is intensity associated with that vector. The four-element row vector is the first row of rotated polarizer's Mueller matrix. However if only relative quantities are of concern, such as degree of polarization or azimuth of the ellipse, constant factors cancel out. 


\subsection{1/qirt.2016.008}
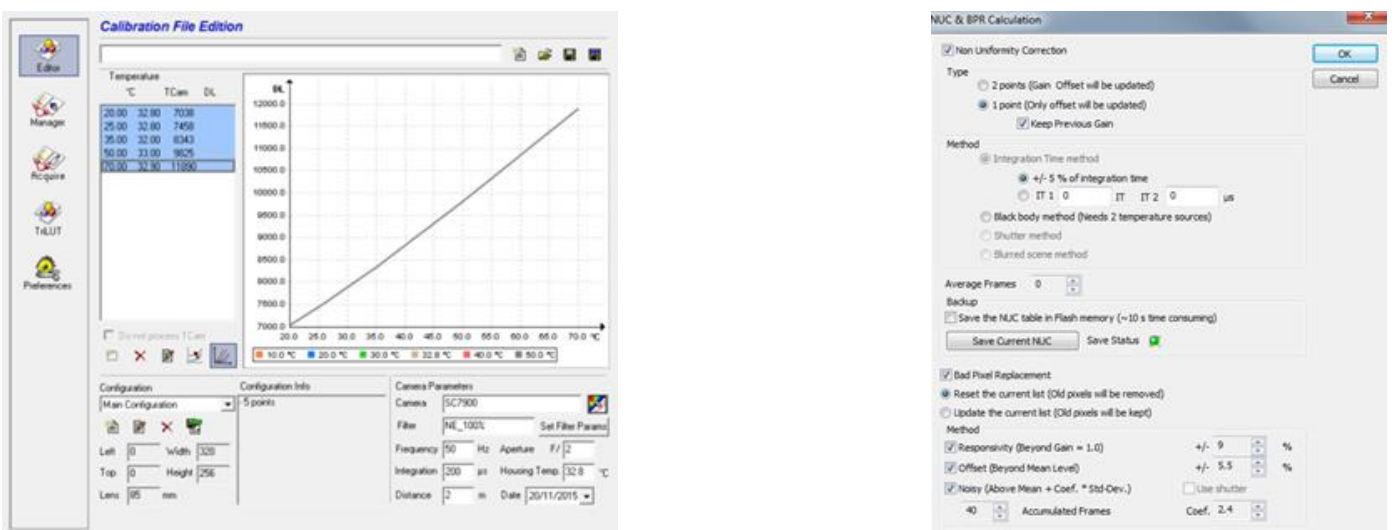

Fig. 4. Calibration (left) and non-uniformity correction (right) windows in Altair software

Like any other method requrining superposition of images, polarimetric imaging is very sensitive to image misalignment. The matching was performed automatically in dedicated software employing gradient-based geometrical matching algorithm with Sum-of-Squared-Differences optimization metric. This algorithm searches for shift in the images that produces lowest SSD result for two measured infrared images. Using a single objective and single FPA for polarimetric image registration makes the registration mono-modal so there is no need for image scale matching, only rigid motion is required to correctly match images. The process of geometrical image calibration is shown on figure 5 . The geometrical matching was performed for every set of image registration comprising four registration with different polarizer angle.
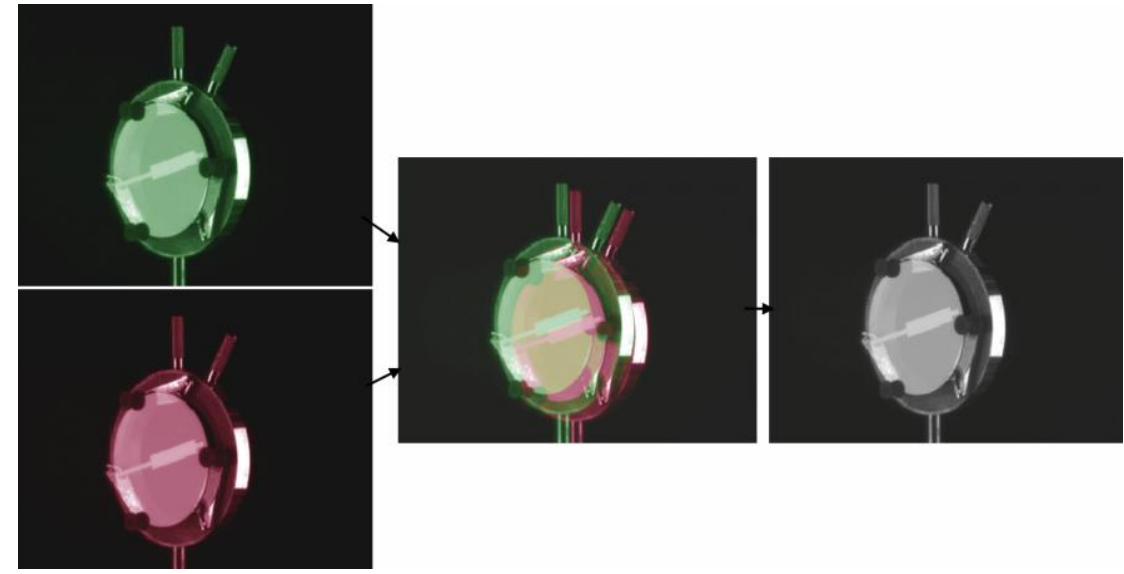

Fig. 5. Superposition of images for polarimetric signature calculation

\subsection{Data presentation}

In order to present multidimensional data in a flat image certain rules of transformation have to be applied to the data. With measured Stokes parameters and derivation of the degree of linear polarization and azimuth of the ellipse a false color data fusion was adapted to visualize measurement results. In visualization method used hue represents an azimuth of the ellipse and color saturation represents degree of linear polarization. This color information is superimposed on gray-level thermal image. This visualization was made by mapping polarimetric data in a linear fashion to a HSV (Hue Saturation Value) color space what is described by equation (7):

$$
\left[\begin{array}{c}
\mathrm{H} \\
\mathrm{S} \\
\mathrm{V}
\end{array}\right]=\left[\begin{array}{c}
\frac{1}{2} \operatorname{tg}^{-1}\left(\frac{S_{2}}{S_{1}}\right) \\
\sqrt{S_{1}^{2}+S_{2}^{2}} \\
S_{0}
\end{array}\right]
$$

Exemplary image of visualized measurement is shown in figure 6. On figure 6 the infrared polarimetric image of windowpanes is presented. The background photography of the building is also presented to put the measurement results into the context. The windowpane of the building presents strong polarimetric signature visualized in saturated colors on infrared image. The changing hue on the window surface indicates change of polarization azimuth what can be contributed to geometry of the corner window. 


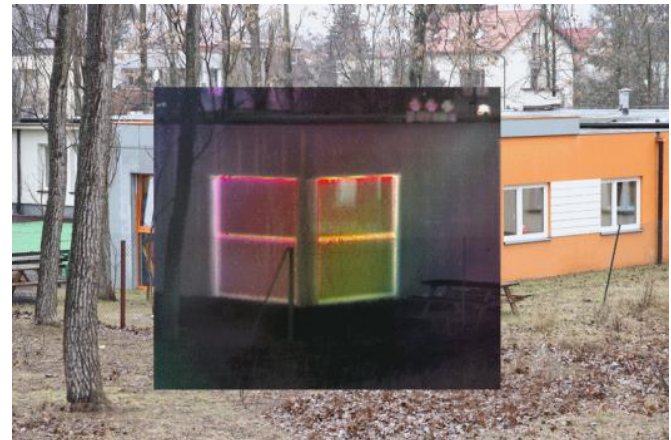

Fig. 6. Visible image with thermal polarimetric signature superimposed

Polarization state of radiation reflected from surface in conditions presented on figure 6 is hard to predict and interpret, because the source of reflected radiation and its geometry is virtually unknown. That is why a measurements of other chosen objects were performed in controlled condition on measurement stand described earlier.

Figure 7 presents objects measured in laboratory: light bulb, plastic, glass bottles and germanium infrared window presented at an angle. All objects were measured in similar environment exhibiting polarized reflections of radiation from warm black bodies placed around the measurement test bench.
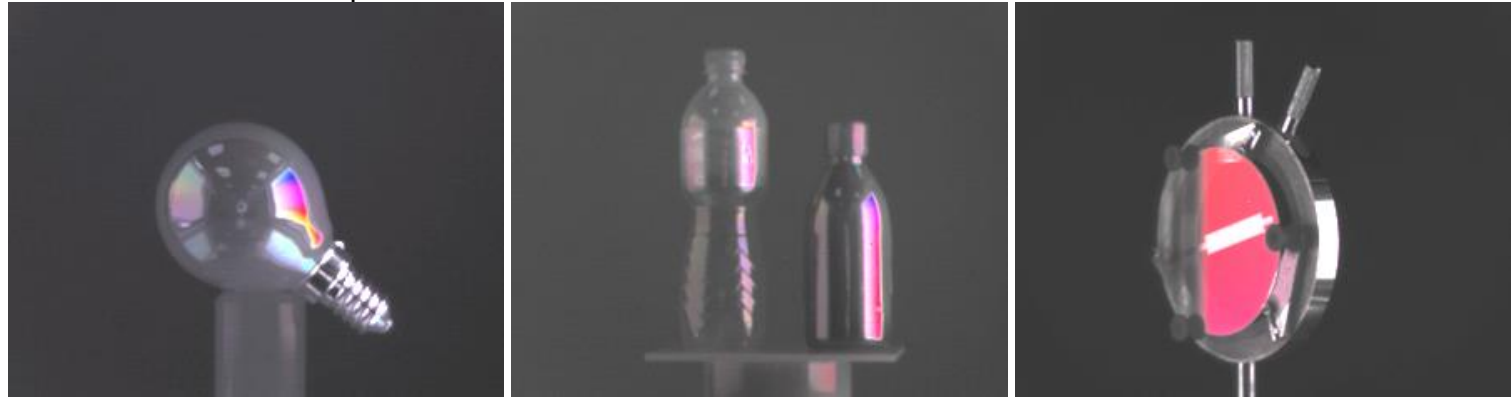

Fig. 7. False-color visualisation of three exemplary measured objects

\subsection{Measurement results}

In order to evaluate possibilities of remote detection using polarization signatures, the polarization radiation content in overall infrared radiation from the scene was evaluated. For this purpose quantitative radiance values of polarized radiation from objects was measured and compared to overall infrared radiation from the scene. For different objects polarization signatures have been obtained and associated radiance values have been calculated. For fast and straightforward quantitative analysis following parameters have been calculated:

mean IR radiance in observed scene $\bar{L}$ :

$$
\bar{L}=E\left\{S_{0}\right\}
$$

where $E\{\}$ is an operator providing frame-wise mean value. The polarized radiance content $\overline{L_{p}}$ of measured scene:

$$
\overline{L_{p}}=E\left\{\sqrt{\mathrm{S}_{1}{ }^{2}+\mathrm{S}_{2}}\right\}
$$

and fraction of polarized radiation in observed scene being a ratio of above values:

$$
R=\frac{\overline{L_{p}}}{\bar{L}}
$$

Table 1. Results of polarised radiation content on chosen representative scenes

\begin{tabular}{|l|l|l|l|}
\hline Scene name & Mean IR radiation $\bar{L}$ & Polarized IR radiation $\overline{L_{p}}$ & Polarized radiation ratio $R$ \\
\hline "LightBulb" & $19.4 \mathrm{~W} / \mathrm{m}^{2} \cdot \mathrm{sr}$ & $0.059 \mathrm{~W} / \mathrm{m}^{2} \cdot \mathrm{sr}$ & $0.31 \%$ \\
\hline "Germanium Plate@55" & $20.2 \mathrm{~W} / \mathrm{m}^{2} \cdot \mathrm{sr}$ & $0.076 \mathrm{~W} / \mathrm{m}^{2} \cdot \mathrm{sr}$ & $0.38 \%$ \\
\hline "Windowpane" & $42.2 \mathrm{~W} / \mathrm{m}^{2} \cdot \mathrm{sr}$ & $0.137 \mathrm{~W} / \mathrm{m}^{2} \cdot \mathrm{sr}$ & $0.33 \%$ \\
\hline "Bottles" & $20.2 \mathrm{~W} / \mathrm{m}^{2} \cdot \mathrm{sr}$ & $0.064 \mathrm{~W} / \mathrm{m}^{2} \cdot \mathrm{sr}$ & $0.32 \%$ \\
\hline
\end{tabular}




\subsection{1/qirt.2016.008}

Resulting radiance parameters of measured scenes are presented in table 1 . Since these values are calculated on a frame-by-frame basis, the polarized radiation ratio $\mathrm{R}$ depends not only on relative polarization (DOLP) but also on a size of polarizing object. The results of this particular specimens were calculated for full frame (320x256) infrared registrations from camera.

Investigation of polarization azimuth based signatures was also carried out. The example picture of color-coded polarization azimuth picture is shown on figure 8. Polarization azimuth of radiation from light bulb was analyzed on its circumference as shown by green arrow on figure. The value of polarization azimuth changes linearly as shown on plot to the right. As expected from geometry of round object, calculated azimuth of polarization ellipse varies in values between \pm 45 degrees
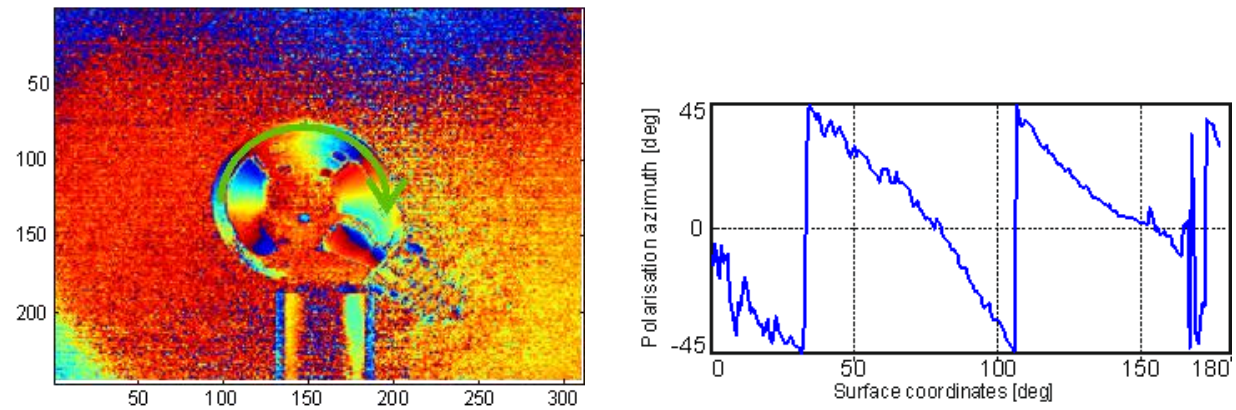

Fig. 8. False-color visualisation of polarization azimuth for object "Lightbulb", profile over upper part of objects" circumference and calculated polarization azimuth for chosen profile

\section{Conclusions}

Polarization state of reflected infrared radiation has been visualized in controlled laboratory conditions. Since polarization state of reflected light depends on mutual orientation of source-object-detector configuration it is usually difficult to predict without sophisticated ray-tracing algorithms. For plane geometries as expected the azimuth of ellipse of polarization is almost constant (germanium window) or varies in a linear fashion (window panes). For curved surfaces the azimuth follows curvature of the surface, notably for a light bulb, profile modulo 90 degrees can be traced.

Absolute radiance values of polarized radiation can be then used as the metrics for remote object detection in non-imaging systems. The spatial distribution of radiation polarization state from object can serve as the signature for automatic image based detection. In the cases where absolute polarized radiation power is weak, the azimuth of polarization signatures can be still of grate value, like in the cases of characteristic azimuth transients in circular objects (light bulb) or well defined flat surfaces. Although radiometric quantities have been provided there is still a need for proper polarimetric comparison with calculated model. For future work comparison will be conducted against a flat and spherical polarization standard artifact.

Results of this scientific work was funded by the National Research and Development Centre under the LIDER program number LIDER/015/479/L-4/12/NCBR/2013.

\section{REFERENCES}

[1] J. Scott Tyo, Dennis L. Goldstein, David B. Chenault, and Joseph A. Shaw "Review of passive imaging polarimetry for remote sensing applications" August 2006 Vol. 45, No. 22 APPLIED OPTICS

[2] Michael W. Kudenov, J. Larry Pezzaniti, Grant R. Gerhart, "Microbolometer-infrared imaging Stokes polarimeter", Proc SPIE 0091-3286 June 2009/Vol. 48(6)

[3] John S. Harchanko, Larry Pezzaniti, David Chenault, Graham Eades Comparing a MWIR and LWIR polarimetric imager for surface swimmer detection Proc. of SPIE Vol. 6972697211 -1

[4] Bruno Lafrance, Maurice Herman "Correction of the Stratospheric Aerosol Radiative Influence in the POLDER Measurements" IEEE TRANSACTIONS ON GEOSCIENCE AND REMOTE SENSING, VOL. 36, NO. 5, SEPTEMBER 1998

[5] B. A. Hooper, B. Baxter, C. Piotrowski, J. Z. Williams and J. "Dugan An Airborne Imaging Multispectral Polarimeter (AROSS-MSP)" Areté Associates IEEE 0-933957-38-1/09

[6] E. F. Stocker, C. Koblinsky, "Sea-surface salinity: the missing measurement," Proceedings of SPIE, Vol. 4881, Crete, Greece, Sept. 23-27, 2002

[7] G.C. Giakos, S. Marotta, C. Narayan, J. Petermann, S. Sestra, D. Pingili, S. A. Tsokaktsidis, D.B. Sheffer, W. Xu, M. Zervakis, G. Livanos, M. Kounelakis "Near Infrared Light Interaction with Lung Cancer Cells" IEEE 9781-4244-7935-1/11

[8] Sławomir Drobczyński "Polarymetria obrazowa z częstością nośną i możliwości jej zastosowania w biomedycynie" Rozprawa doktorska pod kierunkiem prof. dr hab. Henryka Kasprzaka, Wrocław 2006

[9] Firooz Sadjadi, "Electro-Optical Systems for Image Recognition" IEEE 0-7803-71 05-4/01

[10] Born, M., Wolf, E, [Principles of optics], Pergamon Press, (1959) 